\title{
Análise da mortalidade por tétano neonatal no Brasil (1979-1987)
}

\author{
A nalysis of neonatal tetanus mortality \\ in Brazil (1979-1987)
}

Joyce Mendes de Andrade Schramm 1

Odécio Sanches 2

Célia Landman Szwarcwald 3

\footnotetext{
1 Departamento de Epidemiologia e Métodos Quantitativos, Escola Nacional de Saúde Pública, Fundação Oswaldo Cruz. Rua Leopoldo Bulhões 1480, Rio de Janeiro, RJ, 21041-210, Brasil. 2 Instituto de Saúde, Secretaria Estadual de Saúde do Estado de São Paulo. Rua Santo Antônio 590, São Paulo, SP, 01314-000, Brasil. 3 Departamento de Informações para a Saúde, Centro de Informação Científica e Tecnológica, Fundação Oswaldo Cruz. Av. Brasil 4365 , Rio de Janeiro, RJ, 21045-900, Brasil.
}

Abstract This study reports mortality from neonatal tetanus in Brazil (1979-1987), based on an analysi s of death certifi cates. The distribution of causes of death in time and space was used as an indicator of proportional neonatal mortality from tetanus. Underreporting remains a serious problem, particularly in northern and northeastern Brazil. In most municipalities, only one death was notified during the period under consideration, thus highlighting the importance of considering the theoretical approach of "sentinel events" in the control of this di sease. A proportional decrease in mortality from neonatal tetanus was observed in the states of Rondônia, Pará, Sergi pe, São Paulo, Paraná, and Rio Grande do Sul. It was not possible to detect an association between immunization coverage with two doses of tetanus toxoid and neonatal tetanus mortality. The reduction in the number of deliveries taking place in the home during the period studied appears to have played an important role in the reduction of neonatal deaths attributable to this cause.

Key words Neonatal Mortality; Tetanus; Child Health; Mortality; Epidemiology

Resumo Estetrabalho relata a situação da mortalidadepor tétano neonatal no Brasil (1979-1987), baseado na anál ise de registros de óbito. A distribui ção das causas de óbito no tempo eno espaço foi usada como um indicador da mortalidade neonatal proporcional por tétano. A subnotificação permanece um importante problema, principalmente nas regiões norte e nordeste do Brasil. Na maioria dos municípios, apenas um óbi to foi notificado no período, chamando atenção para a importância de selevar em consi deração a abordagem teórica de "evento sentinela" no controle desta enfermidade. Verificou-se redução do indicador de mortalidade proporcional por tétano neonatal nos Estados de Rondônia, Pará, Sergi pe, São Paulo, Paraná, and Rio Grande do Sul. Não foi possível detectar associação entre cobertura vacinal com duas doses de toxói de tetânico e mortali dade proporcional por tétano neonatal. A diminui ção do número de partos domiciliares durante o período de estudo parece ter exercido influência importante na redução das mortes neonatais atribuíveis a esta causa.

Palavras-chave Mortalidade Neonatal; Tétano; Saúde da Criança; Mortalidade; Epidemiologia 


\section{A doença e sua epidemiologia}

O tétano neonatal é uma doença conhecida desde a antigüidade. Transmitida pelo Clostridium tetani, bacilo de natureza anaeróbica de grande resistência, é uma doença que ocupa um lugar especial entre as enfermidades infecciosas. Não é uma doença contagiosa e pode ser considerada como risco ambiental para os seres humanos expostos ao meio contaminado (Cvjetanovic, 1973; Veronesi, 1976; Hill et al., 1992). Estudos realizados em diferentes países mostraram que cerca de $80 \%$ a $90 \%$ dos casos apresentam os primeiros sintomas entre o terceiro e 14o dias (Cvjetanovic, 1982; MSPAS, 1989; Galazka \& Stroh, 1986). Os casos evoluem muito rapidamente para a morte, que vai ocorrer dentro do período neonatal, isto é, o período que compreeende os primeiros 28 dias após o nascimento (Galazka, 1985; Stanfield \& Galazka, 1985; Galazka \& Stroh, 1986; Olusegun \& Parakoyi, 1991).

O tétano neonatal é uma das principais causas da mortalidade neonatal em certos países subdesenvolvidos e, em algumas situações, pode representar cerca de $30 \%$ a $50 \%$ destas mortes (Stanfield \& Galazka, 1984; Galazka \& Stroh, 1986; Melgaard et al., 1988). Sua ocorrência está intimamente relacionada com as condições sócio-econômicas. Altas incidências são encontradas nas camadas pobres da população que não possuem acesso aos serviços de saúde obstétricos e pré-natais e, sobretudo, entre as crianças nascidas de partos domiciliares (Conyer et al., 1991; Cvjetanovic, 1973; Meneghel, 1988; WHO, 1988). Fatores relacionados às condições de higiene do parto estão presentes nas práticas das parteiras e curiosas e são consi derados como fatores de risco que estão associados com o surgimento da doença (WHO, 1982, 1989, 1990).

A letalidade do tétano neonatal é al ta e suas taxas podem variar de $40 \%$ a $90 \%$ de acordo com início dos sintomas, existência e qualidade dos serviços de saúde. É uma das doenças transmissíveis mais subnotificadas. Esta peculiaridade se deve ao fato de o tétano neonatal ser uma doença cuja morte ocorre principalmente nos primeiros sete dias de vida, muitas vezes antes que a criança seja registrada (Boletin Informativo Pai, 1991). Um segundo aspecto relacionado com a subnotificação da doença é que, em algumas culturas, a morte do recém-nascido é ocultada no seio das famílias (Galazka \& Stroh, 1986). Segundo Bytchenko (1973), a situação de subnotificação da doença seria tão grave, que os japoneses se referem à epidemia do tétano como "peculiar quietness".
Para o autor, a epidemia do tétano neonatal não preenche os leitos hospitalares, não sensibilizando assim os serviços e as autoridades para o problema (WHO, 1989).

No Brasil, não se conhece com exatidão a dimensão do problema. Pelas características do País, imagina-se que muitas áreas possam ser classificadas como "zonas produtivas silenciosas". A Secretaria Estadual do Ceará, analisando os dados do Estado para o período de 1985-1990 e aplicando as taxas de incidência do Estado para os municípios do interior, estimou um sub-registro de $90 \%$ dos casos para o Estado (SES, 1991). Inquérito realizado no Município de Nonoai, no Rio Grande do Sul, estimou uma subnotificação de $50 \%$. Foi encontrado um coeficiente de incidência de 0,88/ 1000, não sendo considerado pelos autores como evento raro na região (Meneghel, 1988; Meneghel et al., 1988).

O presente trabalho pretende caracterizar o comportamento da mortalidade por tétano neonatal no Brasil durante o período de 19791987. Assim foram analisadas a distribuição dos óbitos no período por Unidade da Federação e a tendência dos óbitos notificados. Utilizando-se como indicador a mortalidade proporcional por tétano, discute-se a sua evolução temporal frente ao comportamento da cobertura vacinal de gestantes com duas doses de toxóide tetânico e da proporção de partos domiciliares em cada Unidade da Federação.

\section{Comportamento da notificação \\ do tétano neonatal no período}

Na Tabela 1, encontra-se apontado por Unidade Federada o número de municípios que notificaram pelo menos um óbito por tétano neonatal entre 1979 e 1987. Foram utilizadas como fontes de dados as tabulações elaboradas pelo Ministério da Saúde (MS 1990, 1991b) e dados do Censo Demográfico de 1991 (FIBGE, 1991).

Dos municípios totais do País, em 1991, somente $28,2 \%$ notificaram óbitos por tétano neonatal. A distribuição da proporção de municípios que notificaram óbitos por região foi a seguinte: $38,3 \%$ para Região Norte; $20,1 \%$ para Região Nordeste; 29,3\% para Região Sudeste; 30,1\% para Região Sul; 40,7\% para Região Centro-Oeste. Comparando as Regiões Nordeste e Sudeste, chama a atenção que o número de municípios que notificaram óbitos por tétano neonatal no Nordeste é aproximadamente uma vez e meia menor. Porém, é importante observar que estas duas regiões possuem quase o mesmo número de municípios. 
A Figura 1 apresenta o comportamento temporal do número de municípios que notificaram óbitos para cada Região e para todo o País. Observamos um padrão de redução apontado para toda a Federação no referido período, que se deu principalmente devido às Regiões Sul e Sudeste. Não foi possível mostrar o mesmo padrão para as Regiões Norte e Nordeste.

\section{Comportamento dos óbitos} por tétano neonatal

Na Tabela 2, encontra-se a freqüência de municípios segundo o número de óbitos por tétano neonatal registrados entre 1979-1987. Do total de municípios do País, 72\% não notificaram óbitos no referido período. A princípio, estes municípios deveriam ser classificados como zonas silenciosas, não sendo possível definir, pela falta de informação, se seriam zonas silenciosas produtivas ou não produtivas. Já entre os municípios com registro de mortes por tétano neonatal, $51 \%$ notificaram somente um óbito em todo o período. Assim é que, para o País, os valores assumidos pelas moda e mediana da distribuição do número de óbitos foram iguais a 1. Nota-se que, no período, quatro municípios apresentaram um quantitativo de óbitos muito maior que o resto da distribuição: Salvador (90); Fortaleza (98); São Luís (115); Belém (125).

Na Tabela 3, estão calculadas as modas, as medianas da distribuição do número de óbitos, segundo os municípios que notificaram óbitos, e as médias calculadas, incluindo-se e excluindo-se os óbitos das capitais das respectivas Unidades Federadas. Comparando as medidas estatísticas apresentadas na tabela, o estado que se mostrou mais aberrante quanto à distribuição da mediana, foi o Estado de Rondônia, cujo valor encontrado foi 12 . Segundo a observação das duas últimas colunas da tabela, verifica-se que os indicadores referem o peso importante que tiveram os óbitos por tétano neonatal nas capitais dos estados da Região Norte e Nordeste. Por exemplo, nos Estados de Roraima e Amapá, somente as capitais notificaram óbitos. A única exceção foi o Estado de Rondônia, cujo peso dos óbitos não se deu por aqueles registrados na capital, Porto Velho, tal como ocorreu nas Regiões Sudeste e Sul do País.
Tabela 1

Número total de municípios e municípios que notificaram óbitos por tétano neonatal segundo Unidade da Federação. Período: 1979-1987.

\begin{tabular}{|c|c|c|c|c|}
\hline \multicolumn{2}{|c|}{ Estados e regiões } & \multirow{2}{*}{$\begin{array}{l}\text { Municípios } \\
\text { do país }(x) \\
23\end{array}$} & \multirow{2}{*}{$\begin{array}{l}\text { Municípios que } \\
\text { notificaram óbitos }(x) \\
15\end{array}$} & \multirow{2}{*}{$\begin{array}{c}\text { Percentual } \\
(x / y) .100 \\
65,2\end{array}$} \\
\hline Norte & RO & & & \\
\hline & $A C$ & 12 & 6 & 50,0 \\
\hline & AM & 62 & 16 & 25,8 \\
\hline & $\mathrm{RR}$ & 8 & 1 & 12,5 \\
\hline & PA & 105 & 45 & 42,8 \\
\hline & AP & 9 & 1 & 11,1 \\
\hline Total & & 219 & 84 & 38,3 \\
\hline \multirow[t]{9}{*}{ Nordeste } & $\mathrm{MA}$ & 125 & 17 & 13,6 \\
\hline & $\mathrm{PI}$ & 118 & 8 & 6,7 \\
\hline & CE & 178 & 18 & 10,1 \\
\hline & RN & 152 & 11 & 7,2 \\
\hline & PB & 170 & 13 & 7,6 \\
\hline & PE & 168 & 40 & 23,8 \\
\hline & $A L$ & 97 & 34 & 35,0 \\
\hline & SE & 74 & 17 & 22,9 \\
\hline & BA & 303 & 121 & 40,0 \\
\hline Total & & 1385 & 279 & 20,1 \\
\hline \multirow[t]{4}{*}{ Sudeste } & MG & 723 & 258 & 35,6 \\
\hline & ES & 67 & 35 & 52,2 \\
\hline & $\mathrm{RJ}$ & 70 & 43 & 61,4 \\
\hline & SP & 572 & 84 & 14,6 \\
\hline Total & & 1432 & 420 & 29,3 \\
\hline \multirow[t]{3}{*}{ Sul } & PR & 323 & 144 & 44,5 \\
\hline & $\mathrm{SC}$ & 217 & 48 & 22,1 \\
\hline & RS & 333 & 71 & 21,3 \\
\hline Total & & 873 & 263 & 30,1 \\
\hline Centro- & MS & 72 & 55 & 76,4 \\
\hline \multirow[t]{3}{*}{ Oeste } & $\mathrm{MT}$ & 95 & 29 & 30,5 \\
\hline & GO & 290 & 97 & 33,4 \\
\hline & DF & - & 5 & - \\
\hline Total & & 457 & 186 & 40,7 \\
\hline Brasil & & 4366 & 1232 & 28,2 \\
\hline
\end{tabular}

Fonte: Ministério da Saúde, dados a partir de listagem. FIBGE: Dados preliminares do censo, 1991.

A nálise temporal do indicador da mortalidade neonatal proporcional por tétano

Relatórios do Ministério da Saúde apresentam a situação do tétano neonatal entre 1979 e 1987 (MS, 1990, 1991b). Neste período, o coeficiente médio de mortalidade por tétano neonatal para o País foi de 0,11/ 1000 menores de um ano e variando de 0,18 em 1979 a 0,06 em 1987. As Regiões Centro-Oeste e Norte apresentaram os maiores coeficientes médios de mortalidade do País: 0,25/1000 menores de um ano e $0,27 / 1000$ menores de um ano respectivamente, isto é, aproximadamente duas vezes o coeficiente médio nacional. A Região Nordeste apresentou um coeficiente médio de 0,10/1000 menores de um ano que é menor do que o coeficiente médio encontrado para o País. Os valo- 
Figura 1

Número de Município que notificaram óbitos por região e para o país, por cada ano de período estudado.

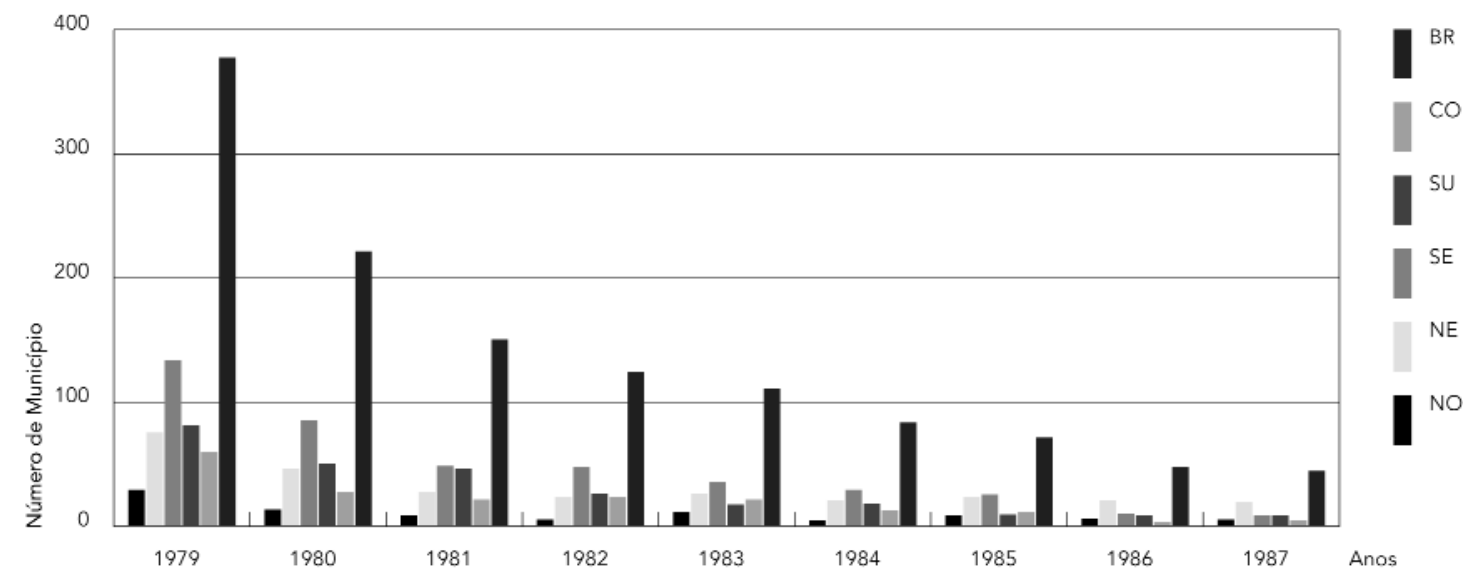

res encontrados para as Regiões Sudeste e Sul foram de 0,08/ 1000 menores de um ano e de 0,11/ 1000 menores de um ano respectivamente (MS, 1990; MS, 1991b). Como valores muito baixos da taxa de mortalidade podem indicar um grau significativo de subnotificação, optouse, neste trabalho, por analisar a evolução da mortalidade neonatal proporcional por tétano. Sendo assim, utilizou-se para a análise temporal do tétano neonatal um procedimento de regressão linear, tendo como variável resposta a mortalidade neonatal proporcional por tétano no período referido e como independente o ano de observação do indicador. Analogamente foram também realizadas as análises temporais dos indicadores de cobertura vacinal e partos domiciliares, utilizando-se as estatísticas de mortalidade (MS, 1982, 1983, 1984, 1985, 1987a, 1987b, 1988, 1991a, 1992) e estatísticas do registro civil (FIBGE, 1979, 1980, 1981, 1982, 1983, 1984, 1985, 1986, 1987), além de tabulações especiais do Ministério da Saúde para 1990 e 1991 (dados não publicados).

Para os procedimentos estatísticos utilizouse a transformação raiz quadrada da mortalidade neonatal proporcional por tétano. Esta transformação foi realizada a fim de estabilizar a variância, como sugerido por Armitage (1974) e Kleinbaum et al. (1988).

Na Tabela 4, encontram-se os resultados da análise temporal deste indicador no período de 1979-1987, por Unidade da Federação, assim como para as variáveis cobertura vacinal e proporção de partos domiciliares. Observou-se que só foi possível apontar redução do indicador para os Estados de Rondônia, Pará, Sergipe, Espírito Santo, São Paulo, Paraná e Rio Grande do Sul. O Estado de Rondônia foi o que apresentou a maior taxa de variação anual média $(-2,07 \%)$ e a maior redução do indicador no período. Poucas Unidades da Federação apresentaram significativos $(p<0,05)$ aumentos de cobertura vacinal com duas doses de toxóide tetânico, como foi o caso de Rondônia, Santa Catarina, Mato Grosso do Sul, Goiás e Distrito Federal. Embora estes aumentos tenham sido constatados, deve ser marcado que os níveis de cobertura vacinal no País ainda podem ser considerados baixos, pois não ultrapassaram $39 \%$ no período, sendo que a cobertura vacinal média para o País no período foi cerca de $21 \%$.

A variável proporção de partos domiciliares apresentou-se com taxas de variação anuais negativas significantes em quase todos os estados da Federação, com exceção dos Estados do Amazonas, Roraima e Maranhão. O Estado de Rondônia apresentou a maior taxa de variação anual do País (-5,18\%) para o período estudado.

\section{Discussão e conclusões}

A subnotificação de óbitos neonatais ainda permanece como um dos problemas de grande relevância no Brasil, tendo sido presenciada em diversas situações durante a elaboração deste trabalho. Assim, comparando-se as Regiões Nordeste e Sudeste, que têm aproximadamen- 
te o mesmo número de municípios, observa-se que é na segunda que se encontra o maior número de municípios que notificaram óbitos por tétano neonatal. Explicações para este resultado recaem no sub-registro de óbitos diferenciado por região. É conhecido que a Região Nordeste possui maiores índices de subnotificação que a Região Sudeste (Moreira, 1986). O sub-registro de óbitos também é diferenciado por idade; na realidade, está inversamente relacionado com a mesma. Portanto, o sub-registro de mortalidade neonatal seria maior que o de mortalidade infantil e assim sucessivamente (Szwarcwald, 1993). A mortalidade do agravo em questão ocorre no período neonatal, sendo que cerca de $90 \%$ desta se dá no período perinatal. Daí acredita-se que o tétano neonatal na região Nordeste estaria amplamente subnotificado, comprovando a subnotificação nas regiões mais pobres do país.

Publicações do Ministério da Saúde apontam que as proporções de municípios cobertos com informação regular de mortalidade variou de $70 \%$ a $75 \%$ no período estudado (MS, 1982, 1983, 1984, 1985, 1987a, 1987b, 1988, 1991b, 1992). Entretanto, Baldijão \& Mello Jorge (1989) apontam coberturas diferentes de municípios com informação regular. Segundo os autores, houve uma redução da cobertura de registro de mortalidade nas Regiões Norte e Nordeste, enquanto nas outras regiões ocorreu o inverso. Isto vem corroborar os nossos achados em relação aos diferenciais regionais de mortalidade por tétano neonatal. A queda da notificação dos óbitos por tétano neonatal no País se deu pela diminuição acentuada ocorrida nas Regiões Sul e Sudeste, onde a doença praticamente desapareceu. É possível que a baixa ocorrência do agravo nessas regiões possa estar restrita a algumas áreas rurais onde ainda exista dificuldade de acesso aos serviços de saúde e onde provavelmente esteja concentrada a subnotificação, como já apontaram Meneghel et al. (1988). Através dos dados utilizados, não nos foi possível dimensionar a subnotificação existente.

Nas Regiões Nordeste e Norte, a concentração de óbitos por tétano neonatal ocorreu predominantemente nas capitais das respectivas Unidades Federadas. É conhecido o fenômeno que ocorre em algumas áreas geográficas denominado “invasão de óbitos". Estas áreas, por apresentarem melhor oferta de serviços de saúde, atrairiam usuários de outras áreas geográficas e os óbitos seriam registrados com erros referentes à procedência do caso. A maior receptividade destas áreas geográficas estaria possivelmente enviesando o registro de óbitos.
Tabela 2

Ó bitos por tétano neonatal ocorridos nos municípios do País. Período de 1979-1987.

\begin{tabular}{|c|c|}
\hline $\begin{array}{l}\text { Número de óbitos } \\
\text { no período }\end{array}$ & $\begin{array}{l}\text { Número de municípios } \\
\text { do País }\end{array}$ \\
\hline 0 & 3.174 \\
\hline 1 & 629 \\
\hline 2 & 238 \\
\hline 3 & 114 \\
\hline 4 & 69 \\
\hline 5 & 38 \\
\hline 6 & 25 \\
\hline 7 & 28 \\
\hline 8 & 11 \\
\hline 9 & 9 \\
\hline 10 & 13 \\
\hline 11 & 9 \\
\hline 12 & 8 \\
\hline 13 & 5 \\
\hline 14 & 2 \\
\hline 15 & 3 \\
\hline 16 & 3 \\
\hline 17 & 4 \\
\hline 18 & 1 \\
\hline 19 & 4 \\
\hline 21 & 1 \\
\hline 22 & 1 \\
\hline 23 & 2 \\
\hline 24 & 1 \\
\hline 27 & 1 \\
\hline 28 & 1 \\
\hline 29 & 1 \\
\hline 30 & 1 \\
\hline 31 & 1 \\
\hline 32 & 1 \\
\hline 35 & 1 \\
\hline 38 & 1 \\
\hline 39 & 1 \\
\hline 44 & 1 \\
\hline 90 & 1 \\
\hline 98 & 1 \\
\hline 115 & 1 \\
\hline 125 & 1 \\
\hline
\end{tabular}

Fonte: Ministério da Saúde, dados fornecidos através de tabulação. 
Tabela 3

Valores da medianas, modas e médias dos óbitos por tétano neonatal segundo municípios que notificaram óbitos no período 1979-1987, segundo Unidade da Federação.

\begin{tabular}{|c|c|c|c|c|}
\hline Estados & Medianas & Modas & $\begin{array}{l}\text { Média por todos } \\
\text { os municípios }\end{array}$ & $\begin{array}{l}\text { Média pelos municípios, } \\
\text { excluindo-se a capital }\end{array}$ \\
\hline RO & 12 & 1 & 14,2 & 14,1 \\
\hline$A C$ & 1 & 1 e 2 & 4,8 & 1,6 \\
\hline$A M$ & 1 & 1 & 4,6 & 2,2 \\
\hline $\mathrm{RR}$ & - & - & 6,0 & 0 \\
\hline PA & 2 & 1 & 5,4 & 2,7 \\
\hline AP & - & - & 24,0 & 0 \\
\hline MA & 1 & 1 & 8,4 & 1,6 \\
\hline PI & 1 & 1 & 2,6 & 1,1 \\
\hline CE & 1 & 2 & 6,8 & 1,4 \\
\hline RN & 1 & 1 & 2,1 & 1,1 \\
\hline PB & 2 & 1 & 2,3 & 1,7 \\
\hline PE & 1 & 1 & 3,1 & 2,4 \\
\hline $\mathrm{AL}$ & 1 & 1 & 2,4 & 1,7 \\
\hline SE & 1 & 1 & 3,5 & 1,7 \\
\hline BA & 2 & 1 & 4,7 & 2,9 \\
\hline MG & 2 & 1 & 2,3 & 2,8 \\
\hline ES & 3 & 1 e 3 & 4,0 & 4,2 \\
\hline RJ & 2 & 1 & 4,8 & 3,8 \\
\hline SP & 1 & 1 & 1,3 & 1,2 \\
\hline$P R$ & 2 & 1 & 2,2 & 2,2 \\
\hline SC & 1 & 1 & 1,5 & 1,5 \\
\hline RS & 1 & 1 & 1,9 & 1,8 \\
\hline MS & 3 & 1 & 4,6 & 4,4 \\
\hline MT & 2 & 1 e 2 & 3,6 & 3,3 \\
\hline GO & 2 & 1 & 2,7 & 2,6 \\
\hline DF & 1 & 1 & 2,2 & 3,3 \\
\hline
\end{tabular}

Fonte: Ministério da Saúde, dados a partir de tabulação.

Obs: Nos Estados do Amapá e Roraima somentes as capitais notificaram óbitos. Para o DF, a média foi calculada para as cidades satélites.
A distribuição dos óbitos por tétano neonatal mostrou que cerca da metade dos municípios que notificaram óbitos apresentaram somente um óbito no período. Sendo o tétano neonatal uma doença de alta letalidade, e podendo a morte ser evitada com uma melhor cobertura e qualidade da atenção pré-natal e dos programas de vacinação, sugere-se que a ocorrência de somente um caso seja considerada como "evento sentinela" na avaliação dos referidos programas (Rutstein, 1976) e particularmente para o programa de vacinação como oportunidade perdida com toxóide tetânico das mulheres em idade fértil (Conyer, 1991; MSPAS, 1989).

A busca de uma explicação para uma possível queda do indicador da mortalidade neonatal proporcional por tétano mostrou-se problemática, na medida em que a análise deste, em relação ao comportamento da cobertura vacinal com duas doses de toxóide tetânico e em relação aos partos domiciliares, não apresentou significância estatística como seria esperado, isto é, o aumento da cobertura vacinal com duas doses de toxóide tetânico e a queda dos partos domiciliares deveriam produzir um impacto importante no referido indicador. Entre os estados que apresentaram redução da mortalidade neonatal proporcional por tétano, o Pará apresentou inclusive uma diminuição da cobertura vacinal. É possível que a qualidade dos dados seja questionável, sobretudo aqueles utilizados nos cálculos das coberturas que envolvem estimativas populacionais nem sempre realizadas com acurácia (Fiocruz, 1989), o que explicaria a ausência de associação com este fator.

Os partos domi ciliares sofreram uma grande redução no País no período 1979-1987, e nas Regiões Sudeste e Sul tal redução ocorreu sobretudo nos municípios do interior, os quais já apresentavam proporções baixas. Acredita-se que a redução do indicador da mortalidade proporcional pode estar relacionada com a redução importante dos partos domiciliares no País. 
Resultados das regressões do indicador da mortalidade neonatal proporcional por tétano no período de 1979-1987 (a) em relação à cobertura vacinal com duas doses de toxóide tetânico (b) e à proporção de partos domiciliares (c).

\begin{tabular}{|c|c|c|c|c|c|c|}
\hline Estados & $\begin{array}{l}\text { Taxa de variação } \\
\text { anual (a) }\end{array}$ & $p$-valor & $\begin{array}{l}\text { Taxa de variação } \\
\text { anual (b) }\end{array}$ & $p$-valor & $\begin{array}{l}\text { Taxa de variação } \\
\text { anual (c) }\end{array}$ & $p$-valor \\
\hline RO & $-2,07$ & $0,01^{*}$ & 23,46 & $0,02 *$ & $-5,18$ & $0,01 *$ \\
\hline$A C$ & $-0,34$ & 0,12 & $-2,45$ & 0,96 & $-2,18$ & $0,03 *$ \\
\hline$A M$ & $-0,48$ & 0,11 & 12,00 & 0,57 & $-1,25$ & 0,15 \\
\hline $\mathrm{RR}$ & $-1,18$ & 0,07 & $-46,68$ & $0,04 *$ & $-5,70$ & 0,16 \\
\hline PA & $-0,56$ & $0,03 *$ & $-16,59$ & 0,23 & $-1,09$ & $0,05^{*}$ \\
\hline$A P$ & $-1,15$ & 0,08 & $-19,96$ & 0,06 & $-1,23$ & $0,01^{*}$ \\
\hline MA & 0,73 & 0,48 & 32,62 & 0,08 & $-0,54$ & 0,28 \\
\hline $\mathrm{PI}$ & 0,54 & 0,79 & 24,50 & 0,07 & $-1,52$ & $0,04 *$ \\
\hline$C E$ & $-0,07$ & 0,89 & 24,22 & 0,30 & $-1,59$ & $0,02 *$ \\
\hline RN & $-0,05$ & 0,69 & 6,38 & 0,26 & $-1,96$ & $0,01 *$ \\
\hline PB & $-0,01$ & 0,18 & 3,34 & 0,86 & $-2,17$ & $0,01 *$ \\
\hline $\mathrm{PE}$ & $-0,04$ & 0,30 & 9,65 & 0,48 & $-1,03$ & $0,01 *$ \\
\hline $\mathrm{AL}$ & $-0,30$ & 0,07 & 15,73 & 0,31 & $-2,23$ & $0,01 *$ \\
\hline SE & $-0,14$ & $0,01^{*}$ & 18,06 & $0,01 *$ & $-2,29$ & $0,01^{*}$ \\
\hline $\mathrm{BA}$ & $-0,28$ & 0,12 & 7,80 & 0,66 & $-1,66$ & $0,01^{*}$ \\
\hline M G & $-0,30$ & 0,16 & 12,83 & 0,53 & $-1,49$ & $0,01 *$ \\
\hline ES & $-0,70$ & $0,01 *$ & 41,65 & $0,01^{*}$ & $-1,19$ & $0,01 *$ \\
\hline RJ & $-0,46$ & 0,12 & 14,79 & 0,24 & $-1,27$ & $0,01^{*}$ \\
\hline SP & $-0,24$ & $0,01 *$ & 0,47 & 0,92 & $-0,39$ & $0,01 *$ \\
\hline$P R$ & $-0,88$ & $0,01 *$ & 35,08 & 0,46 & $-2,33$ & $0,01 *$ \\
\hline SC & 0,31 & 0,12 & 26,13 & $0,01^{*}$ & $-1,70$ & $0,01 *$ \\
\hline RS & $-1,15$ & $0,03 *$ & 0,83 & 0,22 & $-1,40$ & $0,01 *$ \\
\hline MS & $-0,36$ & 0,10 & 47,67 & $0,01^{*}$ & $-2,57$ & $0,01 *$ \\
\hline MT & $-0,06$ & 0,93 & 17,05 & 0,22 & $-2,27$ & $0,01 *$ \\
\hline GO & $-0,52$ & 0,08 & 24,61 & $0,01 *$ & $-2,54$ & $0,01 *$ \\
\hline DF & $-0,11$ & 0,46 & 24,13 & $0,01 *$ & $-1,22$ & 0,06 \\
\hline
\end{tabular}

Fonte: Ministério da Saúde, dados a partir de tabulação.

Ministério da Saúde, Estatísticas de Mortalidade, 1979-1987.

FIBGE, Estatísticas do Registro Civil, 1979-1987.

* Significativo no nível de $5 \%$.

\section{Referências}

ARM ITAGE, P., 1974. Statistical Methods in Medical Research. 3. London: Blackwell Scientific Publications.

BALDIJÃO, M. F. A. \& MELLO JORGE, M. H. P., 1989. Óbitos fetais no Brasil no período de 1980-1985. Centro Brasileiro de Classificação de Doenças. Ministério da Saúde/Universidade de São PauIo/Organização Pan-Americana da Saúde-Organização Mundial da Saúde: Série Divul gação no 4.

BOLETIN INFORM ATIVO PAI, 1991. La Mortalidad por Tétanos Neonatal. OPAS, año 13, no 2.

BYTCHENKO, B., 1973. Tendencias recientes de la mortalidad por tétanos en el mundo. Boletin de la Oficina Sanitaria Panamericana, 75:412-415.
CONYER, R. T.; AMOR, J. S.; IZABA, B. S.; DIAZ, A. T.; CARRILLO, L. L.; MUÑOZ, J. L. C. \& RINCÓN, H. G., 1991. Factores asociados a la mortalidad por tétanos neonatal en el área rural de Jalisco. Salud Pública de México, 33:207-213.

CVJETANOVIC, B., 1973. Epidemiología del tétanos considerada desde un punto de vista práctico de salud pública. Boletín de la Oficina Sanitaria Panamericana, 75:315-24.

CVJETONOVIC, B., 1982. Tetanus-Infection without inter-human transmission. In: The Population Dynamics of Infectious Diseases: Theory and Applications (R. M. Anderson, ed.) pp. 43-47, London/ New York: Chapman and Hall Ltda. 
FIBGE (Fundação Instituto Brasileiro de Geografia e Estatística), 1979. Estatísticas do Registro Civil, v.6.

FIBGE (Fundação Instituto Brasileiro de Geografia e Estatística), 1980. Estatísticas do Registro Civil, v.7.

FIBGE (Fundação Instituto Brasileiro de Geografia e Estatística), 1981. Estatísticas do Registro Civil, v.8.

FIBGE (Fundação Instituto Brasileiro de Geografia e Estatística), 1982. Estatísticas do Registro Civil, v.9.

FIBGE (Fundação Instituto Brasileiro de Geografia e Estatística), 1983. Estatísticas do Registro Civil, v.10.

FIBGE (Fundação Instituto Brasileiro de Geografia e Estatística), 1984. Estatísticas do Registro Civil, v.11.

FIBGE (Fundação Instituto Brasileiro de Geografia e Estatística), 1985. Estatísticas do Registro Civil, V.12.

FIBGE (Fundação Instituto Brasileiro de Geografia e Estatística), 1986. Estatísticas do Registro Civil, V.13.

FIBGE (Fundação Instituto Brasileiro de Geografia e Estatística), 1987. Estatísticas do Registro Civil, v.14.

FIBGE (Fundação Instituto Brasileiro de Geografia e Estatística), 1991. Censo Demográfico 1991.

FIOCRUZ (Fundação Oswaldo Cruz), 1989. Inquéritos populacionais para avaliação de coberturas de vacinação. Dados, 13.

GALAZKA, A. \& STROH, G., 1986. Le Tetanus du Nouveau-né. Directives pour les Enquêtes au Niveau des Communautés sur la Mortalité Imputable du Tetanus Neonatal. Genebra: World Health Organization/Expanded Program on Imunization. (mimeo.)

GALAZKA, A., 1985. Control of neonatal tetanus. Indian Journal of Pediatrics, 52.

HILL, B. G.; OSTERHOUT, S. \& MWILLWT, H. T., 1992. Clostridium. In: Zinsser Microbiology (B. G. Hill, ed.), pp. 636-655. Connecticut: Appleton Century Crofts.

KLEINBAUM, D. G.; KUPPER, L. L. \& MULLER, K. E., 1988. Applied Regression Analysis and other Multivariate Methods. 2a ed., Boston: PWS-Kent Publishing Company.

MELGAARD, B.; MUTIE, D. M. \& KIMANI, G., 1988. A cluster survey of mortality due to neonatal tetanus in Kenya. International Journal of Epidemiology, 17:174-177.

MENEGHEL, S.; DANILEVICZ, N.; KMETZSEH, C. \& VON MUHLEN, D., 1988. Inquérito de morbimortalidade do tétano neonatal no Município de Nonoai, Rio Grande do Sul. Relatório de Pesquisa. (mimeo.)

MENEGHEL, S. N., 1988. Vigilância epidemiológica do tétano no Rio Grande do Sul, Brasil. Boletin de la Oficina Sanitaria Panamericana, 105:139-150.

M OREIRA, M. M., 1986. Registro de óbitos no Nordeste: estimativa da cobertura 1979-1981.Anais do V Encontro Nacional de Estudos Populacionais. São Paulo: Associação Brasileira de Estudos Populacionais.

MS (Ministério da Saúde), 1982. Estatísticas de Mortalidade, Brasil, 1979. Brasília: Centro de Documentação do Ministério da Saúde.

MS (Ministério da Saúde), 1983. Estatísticas de Mortalidade, Brasil, 1980. Brasília: Centro de Documentação do Ministério da Saúde.
MS (Ministério da Saúde), 1984. Estatísticas de Mortalidade, Brasil, 1981. Brasília: Centro de Documentação do Ministério da Saúde.

MS (Ministério da Saúde), 1985. Estatísticas de Mortalidade, Brasil, 1982. Brasília: Centro de Documentação do Ministério da Saúde.

MS (Ministério da Saúde), 1987a. Estatísticas de Mortalidade, Brasil, 1983. Brasília: Centro de Documentação do Ministério da Saúde.

MS (Ministério da Saúde), 1987b. Estatísticas de Mortalidade, Brasil, 1984. Brasília: Centro de Documentação do Ministério da Saúde.

MS (Ministério da Saúde), 1988. Estatísticas de Mortalidade, Brasil, 1985. Brasília: Centro de Documentação do Ministério da Saúde.

MS (Ministério da Saúde), 1990. Programa de Controle de Tétano Neonatal no Brasil. Uma Proposta deAção.

MS (Ministério da Saúde), 1991a. Estatísticas de Mortalidade, Brasil, 1986. Brasília: Centro de Documentação do Ministério da Saúde. (mimeo.)

MS (Ministério da Saúde), 1991b. O Enfoque de Risco na Eliminação do Tétano Neonatal no Brasil. (mimeo.)

MS (Ministério da Saúde), 1992. Estatísticas de Mortalidade, Brasil, 1987. Brasília: Centro de Documentação do Ministério da Saúde.

MSPAS (Ministério de Salud Pública y Asistencia Social), 1989. Tétanos Neonatal. El Salvador: MSPAS.

OLUSEGUN, B. \& PARAKOY, B., 1991. Cluster survey for poliomylites and neonatal tetanus in Illorin, Nigeria. International Journal of Epidemiology, 20:515-520.

RUTSTEIN, D. D.; BERENBERG, W. \& CHALMERS, T. C., 1976. Measuring the quality of medical care, a clinical method. New England Journal of Medicine, 294:582-588.

SES (Secretaria Estadual de Saúde), 1991. Programa de Eliminação do Tétano Neonatal. Ceará:SES

STANFIELD, J. P. \& GALAZKA, A., 1984. Neonatal tetanus in the world today. Bulletin of the World Health Organization, 62:647-669.

STANFIELD, J. P. \& GALAZKA, A., 1985. Neonatal tetanus - an underreprted scourge. World Health Forum, 6:127-129.

SZWARCWALD, C. L., 1993. Estimativas da Mortalidade Infantil como Função da Distribuição Etária dos "bitos Registrados: Proposta de um Procedimento. Tese de Doutorado, Rio de Janeiro: Escola Nacional de Saúde Pública, Fundação Oswaldo Cruz.

VERONESI, R., 1976. Doenças Infecciosas e Parasitárias. Rio de Janeiro: Guanabara Koogan.

WHO (World Health Organization), 1982. Prevention of Neonatal Tetanus. Expanded Programme on Immunization. Weekly Epidemiological Record, 18.

WHO (World Health Organization), 1988. Expanded Programme on Immunization. Weekly Epidemiological Record, 3.

WHO (World Health Organization), 1989. Enquêtes sur la mortalité due au Tétanos Néonatal. Weekly Epidemiological Record, 40.

WHO (World Health Organization), 1990. Enquête sur le Tétanos Néonatal. Weekly Epidemiological Record, 35. 